\title{
A shift from "negative" to "positive" transfer under the A-C paradigm with increased number of C-D control pairs in a mixed list'
}

WILLIAM F, BATTIG

UNIVERSITY OF MARYI IND

Transfer under a standard A-C (first-list stimuli paired with new responses) verbal paired-associate transfer paradigm was found to shift from positive when only two A-C pairs were included with six new C-D control pairs in a mixed list, to increasing negative transfer with four pairs of both types, and with six A-C along with two C-D pairs. Since the latter most closely corres ponds to typical mixed-list transfer conditions, these results are indicative of a spurious overestimate of negative transfer for other paradigms under mixedlist conditions.

Amidst the host of inconsistencies pervading the literature on intertask similarity effects upon transfer (see, e.g., Battig, 1966; Underwood, 1961), there has been widespread agreement and acceptance of (a) new responses paired with old first-list stimuli ( $A-C$ paradigm) as a condition producing consistent and significant negative transfer, and (b) the equivalence of the transfer effects produced by the A-C and other transfer paradigms under mixed- and unmixed-list procedures (Twedt \& Underwood, 1959). Mixed-list transfer comparisons, however, have almost without exception included more than one transfer condition or paradigm in addition to the $C-D$ control paradigm (e.g., Kausler \& Kanoti, 1963; Mandler \& Heinemann, 1956; Merikle \& Battig, 1963; Twedt \& Underwood, 1959), so that the second transfer list has contained fewer new C-D control pairs than pairs containing one or more first-list items. That such conditions may produce spurious negative transfer is suggested by Battig \& Berry's (in press) recent demonstration of a marked facilitation on new $C-D$ pairs (relative to pairs consisting of individual items previously used in a verbal-discrimination or multiple-choice recognition task) when there were fewer new than pretrained pairs in the PA list. This "negative" transfer on pretrained pairs contrasted sharply with the generally positive effects of pretraining when the number of pretrained pairs was equal to or fewer than the number of new pairs. These differential transfer effects were interpreted as reflecting a kind of "isolation" effect, such that "S's attention is attracted to pairs consisting of new previously unseen items when there a re relatively few of these" (Battig \& Berry, in press).

The purpose of the present study was to determine whether variations in the proportion of new C-D pairs in a mixed list may produce similar differential transfer effects under the standard A-C transfer paradigm. Selection of the A-C paradigm for this comparison was based upon the predominant interest in this paradigm in previous transfer research, along with its apparent greater stability than other transfer paradigms under systematic variations in such other factors as meaningfulness (e.g., Merikle \& Battig, 1963).

Method

Each of 36 Ss learned in succession two eight-pair CVC lists, using the recall method with a 4-sec. presentation rate and a 12-sec. intertrial interval. All Ss learned the first list to a criterion of 40 total correct responses summed over all trials, whereas second-list learning was to a criterion of two successive errorless trials.

Three groups of $12 \mathrm{Ss}$ each differed solely as to number of the eight second-list pairs representing A-C and new $C-D$ pairs. Under the 2-6 condition, there were two A-C and six C-D pairs. The $4-4$ condition involved equal numbers (four) of $A-C$ and $C-D$ pairs, whereas the 6-2 condition consisted of six $A-C$ and two $C-D$ pairs. All groups learned the same second list of eight moderate association-value CVC pairs previously shown to be equivalent in rated lea rning difficulty (Battig, 1960), and of minimal intralist similarity. To counterbalance individual second-list pairs with respect to their representation of $A-C$ and $C-D$ paradigms, four different first lists were used under 2-6 and 6-2 conditions, with two first lists under the 4-4 condition. Each A-C first-list pair was constructed by pairing the secondlist stimulus term with a new response CVC of equivalent association value. All remaining first-list pairs were comparable to the second-list pairs in rated learning difficulty (Battig, 1960) but consisted of two CVCs maximally dissimilar to any appearing in the second list.

Each of three Es ran one-third of the Ss under each condition, with an equal number of Ss using each first list within each condition. Assignment of Ss to Es and conditions was unsystematic, based upon a combination of coincidence of free times for Ss and Es with a predetermined different order for each $\mathrm{E}$ specifying assignment of Ss to lists and conditions.

\section{Results}

Total errors to criterion on the first list proved to be closely comparable across the three groups $(F<1)$, and also did not differ significantly between the three Es and the various lists within conditions. Although more total second-list errors were made under the 2-6 
condition (37.5) than the 4-4 or 6-2 conditions (both 31.2), this difference also fell far short of significance $(\mathrm{F}<1)$.

Relative performance on A-C and C-D pairs, however, did differ systematically across the three conditions. Under the 2-6 condition, fewer mean errors per pair were made on $\dot{A}-C(4.38)$ than $C-D$ pairs (4.97), and nine of the 12 Ss showed superior performance on $A-C$ pairs. Although absolute performance on the A-C pairs actually improved slightly with increasing numbers of these under both 4-4 (4.11) and 6-2 (4.10) conditions, C-D performance showed a far greater progressive improvement under 4-4 (3.77) and 6-2 (3.25) conditions. Within each of the latter two groups, eight of the $12 \mathrm{Ss}$ made more errors per pair on A-C than on C-D pairs. The indicated improvement on $C-D$ pairs with decreasing numbers thereof fell short of significance $(p<.10)$, however, even by analysis of covariance based on first-list total errors as a covariate. Likewise, the improvement on $C-D$ relative to $A-C$ pairs from 2-6 to 6-2 conditions did not attain statistical significance $(\mathrm{p} \approx .10)$.

Closer examination of the data for individual Ss indicated the nonsignificance of the differences between conditions to be attributable to a general tendency for those few Ss showing negative A-C transfer in the 2-6 group, and positive $\mathrm{A}-\mathrm{C}$ transfer in the 4-4 and 6-2 groups, to have made significantly more $(p<.025)$ total second-list errors $(44.8)$ than did the remaining Ss (28.9). To eliminate the excessive weighting in the previous analyses of the transfer performance of these few deviant Ss, a percentage transfer measure was calculated for each $\mathrm{S}$ by dividing the difference between C-D and A-C mean errors per pair by the sum of these two values (Murdock, 1957; Formula 3b). This measure showed a highly significant $(\mathrm{p} \approx .005)$ decrease from the $2-6(12.5 \%)$ to $4-4(-14.5 \%)$ and $6-2$ conditions $(-20.3 \%)$. Discussion

In agreement with the findings of Battig \& Berry (in press), the present results clearly show a significant improvement in relative performance on C-D pairs as the number of these included in an eight-pair list decreases from six to two. Thus if new C-D pairs represent the transfer baseline under mixed-list conditions, transfer under the $\mathrm{A}-\mathrm{C}$ paradigm changes from positive when the list consists predominantly of $C-D$ pairs to negative when the latter pairs are equivalent or fewer in number as compared with $\mathrm{A}-\mathrm{C}$ pairs. Since previous mixed-list transfer comparisons have typically included a minority of C-D pairs, it is apparent that amount of negative transfer under other paradigms has been spuriously overestimated as a result of the facilitation of performance on the small number of $C-D$ pairs employed in these mixed-list comparisons.

Further support for the Battig-Berry (in press) interpretation of the facilitation on a minority of $C-D$ pairs as representing a kind of "isolation" effect is provided by the present finding that those few Ss who failed to show facilitated performance on whichever type of pair was in the minority also showed a significant decrement in overall second-list performance as compared with the other Ss. This result further suggests that classification of second-list pairs into two or more types or categories offers an important means whereby Ss can combat interference in pairedassociate learning, particularly for a type of pair representing a minority of the list. Thus besides pointing to a possible source of contamination in mixed-list transfer comparisons, the present results suggest the mixed-list procedure to offer a potentially useful technique for the systematic manipulation of sources of inter- and intralist interference and the investigation of techniques employed by Ss to overcome such interference (Postman, 1963).

\section{References}

Battig, W. F. Comparison of two methods of scaling nonsensesyllable pairs for ease of learning. Psychol. Rep., 1960, 6, 363366.

Battig, W. F. Facilitation and interference. In E. A. Bilodeau (Ed.), Acquisition of skill. New York: Academic Press, 1966 (in press).

Battig, W. F., \& Berry, J. K. Verbal-discrimination and multiplechoice recognition pretraining effects on paired-associate performance. J. exp. Psychol., in press.

Kausler, D. N., \& Kanoti, G. A. R-S learning and negative transfer effects with a mixed list. J. exp. Psychol., 1963, 65, 201-205.

Mandler, G., \& Heinemann, S. H. Effect of overlearning of a verbal response on transfer of training. $J$. exp. Psychol., 1956, 51, 3946.

Merikle, P. M., \& Battig, W. F. Transfer of training as a function of experimental paradigm and meaningfuiness. J. verbal Learn. verbal Behav., 1963, 2, 485-488.

Murdock, B. B., Jr. Transfer designs and formulas. Psychol. Bull., $1957,54,313-326$.

Postman, L. Does interference theory predict too much forgetting? $J$. verbal Learn. verbal Behav., 1963, 2, 40-48.

Twedt, H. M., \& Underwood, B. J. mixed vs. unmixed lists in transfer studies. J. exp. Psychol., 1959, 58, 111-116.

Underwood, B. J. An evaluation of the Gibson theory of verbal learning. In C. N. Cofer (Ed.), Verbal learning and verbal behavior. New York: McGraw-Hill, 1961. Pp. 197-217.

Note

1. This research was supported in part by Public Health Service Research Grant HD-01062 from the National Institute of Child Health and Human Development. The author is also indebted to Sheila L. Hunt, Jon Maiden, and Glen E. Webb, who served as experimenters. 